\title{
Suppressor of cytokine signaling-1 regulates acute inflammatory arthritis and $T$ cell activation
}

\author{
Paul J. Egan, ${ }^{1}$ Kate E. Lawlor, ${ }^{1}$ Warren S. Alexander, ${ }^{2}$ and Ian P. Wicks ${ }^{1}$ \\ ${ }^{1}$ Reid Rheumatology Laboratory, Division of Autoimmunity and Transplantation, and \\ ${ }^{2}$ Division of Cancer and Hematology, Walter and Eliza Hall Institute of Medical Research, Parkville, Victoria, Australia
}

\begin{abstract}
Suppressor of cytokine signaling-1 (SOCS-1) is a negative regulator of cytokine signaling. To investigate the role of SOCS-1 in regulating inflammatory and immune responses in disease, acute inflammatory arthritis was induced in mice lacking SOCS-1. Expression of SOCS-1 protein was detected within synovial granulomas and pannus tissue of WT mice by day 7 following induction of acute arthritis. The severity of synovial inflammation and joint destruction at the peak of disease was greater in the absence of SOCS-1, although disease resolution occurred normally. There was an increased percentage of myeloid cells infiltrating the synovium in mice lacking SOCS-1, and SOCS-1 promoter activity was present in synovial macrophages, lymphocytes, and fibroblasts, but not granulocytes. The $\mathrm{T}$ cell response in draining $\mathrm{LNs}$ was also dysregulated, as popliteal LNs from mice lacking SOCS-1 contained approximately fivefold more cells at the peak of acute arthritis. These cells were hyperproliferative on exposure to antigen in vitro, and purified splenic $\mathrm{CD} 4^{+} \mathrm{T}$ cells from mice lacking SOCS-1 proliferated more strongly in response to stimulation with anti-CD3. Reporter gene expression was detected in $\mathrm{CD}^{+}{ }^{+} \mathrm{T}$ cells bearing the activation markers $\mathrm{CD} 25, \mathrm{CD} 44$, and CD69. SOCS-1 is therefore expressed in hematopoietic and nonhematopoietic cell types in vivo and is an important regulator of acute inflammatory arthritis and of $\mathrm{CD}^{+} \mathrm{T}$ cell activation.
\end{abstract}

J. Clin. Invest. 111:915-924 (2003). doi:10.1172/JCI200316156.

\section{Introduction}

Rheumatoid arthritis (RA) is an autoimmune disease characterized by inflammation, synovial hyperplasia, neoangiogenesis, and progressive destruction of cartilage and bone. Although the etiology of RA is complex, inflammatory cytokines play a central role. Overproduction of inflammatory cytokines in the synovium, particularly TNF- $\alpha$, IL-1, IL- 6 , and GM-CSF, is characteristic of RA, and of various rodent models of the disease, such as collagen-induced arthritis (CIA) and adjuvant-induced arthritis (1). Neutralization of cytokine activity by mAb's or soluble cytokine receptors has been shown to inhibit the establishment and progression of disease (2-5), and TNF- $\alpha$ and IL-1 antagonists are currently in clinical use for the treatment of RA. A significant proportion of RA patients treated with TNF- $\alpha$

Received for publication June 11, 2002, and accepted in revised form January 7, 2003

Address correspondence to: Ian P. Wicks, Reid Rheumatology Laboratory, Division of Autoimmunity and Transplantation, Walter and Eliza Hall Institute of Medical Research, $1 G$ Royal Parade, Parkville, Victoria 3050, Australia. Phone: 61-3-9345-2466; Fax: 61-3-9347-0852; E-mail:wicks@wehi.edu.au.

Conflict of interest: The authors have declared that no conflict of interest exists.

Nonstandard abbreviations used: rheumatoid arthritis (RA); collagen-induced arthritis (CIA); suppressor of cytokine signaling (SOCS); signal transducer and activator of transcription (STAT); Janus kinase (JAK); methylated BSA (mBSA); fluorescein-Dgalactopyranoside (FDG); tritiated thymidine ( $\left.{ }^{3} \mathrm{H}-\mathrm{TdR}\right)$. antagonists, however, fail to respond (6), while mice deficient in TNF- $\alpha$ can still develop severe CIA (7). Compared with inhibition of a single cytokine in a complex disease such as RA, a more effective treatment might be inhibition of the activities of multiple cytokines. One strategy that could accomplish this would be to target shared cytokine signal transduction pathways using the suppressor of cytokine signaling (SOCS) molecules.

The SOCS family of proteins act as negative regulators of cytokine signal transduction (8). The family consists of eight proteins, SOCS- 1 to SOCS-7 and cytokineinducible $\mathrm{SH} 2$-containing protein (CIS), which act to inhibit the signal transducer and activator of transcription (STAT) signal transduction pathway (9). The mechanisms by which SOCSs inhibit STAT-mediated signal transduction vary: while SOCS-1 and SOCS-3 both inhibit Janus kinase (JAK) activity, SOCS-1 binds directly to JAKs with high affinity and inhibits tyrosine kinase activity $(10,11)$. In contrast, SOCS-3 appears to require interactions with receptors, such as gp130, for recruitment to the signaling complex (12). Experiments with cell lines in vitro have shown that SOCS-1 can be induced following stimulation with multiple cytokines that use the JAK-STAT signal transduction pathway, including IL-2, IL-3, IL-6, and IFN- $\gamma$, while overexpression of SOCS-1 can inhibit signaling by numerous other cytokines (8). In addition, SOCS-1 has been shown to inhibit TNF- $\alpha$-mediated apoptosis of fibroblasts by inhibiting signaling through the p38 MAPK pathway (13). SOCS-1 has also been shown to be 
involved in the negative regulation of responses induced by LPS signaling through Toll-like receptor $4(14,15)$. Mouse models of arthritis have revealed important roles for the cytokines IL- 6 and GM-CSF, as mice deficient in either cytokine are less susceptible to CIA (16-18). Both cytokines use the JAK-STAT signal transduction pathway, although the actual molecules used differ: IL-6 signaling predominantly occurs through STAT3 activation, while GM-CSF uses STAT5. In addition, T cell activation and expansion, under the control of cytokines such as IL-2, which signals through phosphorylation of STAT5, are an important component of inflammatory arthritis. Since SOCS-1 can inhibit downstream phosphorylation of both STAT3 and STAT5 $(9,10)$, SOCS-1 might inhibit the activity of multiple cytokines and therefore be an important negative regulator of both inflammation and $\mathrm{T}$ cell activation associated with inflammatory arthritis. We have addressed this issue by examining acute inflammatory arthritis in SOCS-1-deficient mice. Mice lacking SOCS-1 die at 2-3 weeks of age from an inflammatory syndrome characterized by fatty degeneration and necrosis in the liver and monocytic infiltration into numerous organs, including liver, muscle, pancreas, heart, and lungs (19). Lymphoid deficiencies in SOCS-1-deficient mice include reduced thymic cellularity and B cell lymphopenia. Most of the pathological effects seen in SOCS-1-deficient mice are mediated by IFN- $\gamma$, since mice lacking both SOCS- 1 and IFN- $\gamma$ do not exhibit this syndrome and survive until adulthood (20). Despite the ability of SOCS-1 to inhibit signaling of a number of proinflammatory cytokines, the response of these mice in models of inflammatory diseases has not been examined. We have therefore used the SOCS-1/IFN- $\gamma$ double-knockout mouse to examine the effects of SOCS-1 in regulating a model of acute inflammatory arthritis.

Methylated BSA/IL-1 arthritis is induced by intraarticular injection of methylated BSA (mBSA) into the knee, followed by three daily subcutaneous injections of IL-1 $(21,22)$. Inflammatory arthritis peaks 7 days after injection and resolves by day 21 . The advantages of this model are the high incidence of arthritis in injected knee joints and the rapid and reproducible kinetics of disease onset and resolution. Development of arthritis in this model is dependent on $\mathrm{CD} 4^{+} \mathrm{T}$ cells but is independent of B cells and CD8 ${ }^{+} \mathrm{T}$ cells (ref. 22; K.E. Lawlor and I.P. Wicks, unpublished observations). Here we show that the mBSA/IL-1 model is also independent of IFN- $\gamma$, which allowed us to directly examine the effects of SOCS-1 in the development of inflammatory arthritis. We show that arthritis is exacerbated in the absence of SOCS-1, and that SOCS-1 is expressed by several cell types in the arthritic joint and regulates mononuclear cell infiltration in the synovi$\mathrm{um}$ and $\mathrm{T}$ cell proliferation in the draining LNs.

\section{Methods}

Mice. C57BL/6 mice and $I F N-\gamma^{/-}$mice maintained on a C57BL/6 background were obtained from the Walter and
Eliza Hall Institute of Medical Research animal services (Kew, Australia). The SOCS-1 locus was targeted in 129/Sv ES cells as described previously (20). ES cell clones bearing one disrupted SOCS-1 allele were injected into C57BL/ 6 blastocysts, and the chimeric mice generated were bred with C57BL/ 6 mice to generate heterozygotes on a mixed $129 / \mathrm{Sv}$ and C57BL/6 generic background. SOCS-1 $1^{+/-}$mice were then bred with C57BL $/ 6 \mathrm{IFN}-\gamma^{/-}$mice to produce SOCS-1 ${ }^{+/-} I F N-\gamma^{+/-}$compound heterozygotes. These compound heterozygotes were subsequently interbred to produce SOCS-1-/-IFN- $\gamma^{-/}$and SOCS-1 $1^{+/} I F N-\gamma^{--}$ mice as required. Once mice of these individual genotypes were obtained, they were interbred for maintenance as individual lines. All animal procedures were approved by the institutional ethics committee.

Arthritis induction. Induction of arthritis using mBSA and IL-1 was performed as previously described $(21,22)$. Briefly, 6- to 12-week-old mice were anesthetized and injected with $10 \mu \mathrm{l}$ of a $20-\mathrm{mg} / \mathrm{ml}$ solution of mBSA (Sigma-Aldrich, St. Louis, Missouri, USA) in saline, or saline alone, into one or both knee joints. Mice were then injected with $20 \mu \mathrm{l}$ of $12.5 \mu \mathrm{g} / \mathrm{ml}$ recombinant human IL-1 $\beta$ (National Cancer Institute, Bethesda, Maryland, USA) subcutaneously in the footpad. The IL-1 $\beta$ injection was repeated on the next 2 days. Mice were sex-and age-matched within experiments, and at least 5 mice were included in each experimental group. All experiments were repeated at least three times.

Histologic assessment of arthritis. Mice were euthanized on day 7 after mBSA injection, and the knee joints were removed, fixed in $10 \%$ neutral buffered formalin for at least 2 days, decalcified, and processed to paraffin. Four-micrometer frontal tissue sections were cut at four depths approximately $100 \mu \mathrm{m}$ apart and stained with $H \& E$. Arthritis was assessed by an investigator who was blinded to the experimental groups, and five features of arthritis were graded for severity from 0 (normal) to 5 (severe) as previously described (22). The overall mean histologic severity score for an experimental group (maximum of 25) was calculated by averaging the sum of the five histological features, with each feature scored at four section depths.

Immunohistochemistry. Paraffin sections were dewaxed and rehydrated and stained with purified goat polyclonal antibodies specific to the C-terminal region of murine and human SOCS-1 (Santa Cruz Biotechnology Inc., Santa Cruz, California, USA) or purified goat anti-hamster Ig antibodies as a negative control (Caltag Laboratories Inc., Burlingame, California, USA). Primary antibodies were detected with peroxidase-conjugated rabbit anti-goat Ig antibodies (DAKO A/S, Glostrup, Denmark), and staining was visualized with diamino-benzidine as the substrate. Sections were counterstained with hematoxylin.

Isolation of cells from synovial tissue and peritoneal cavity. Synovial tissue was dissected from knee joints of mice following induction of arthritis and digested in a cocktail of $2.4 \mathrm{mg} / \mathrm{ml}$ dispase (Boehringer Mannheim, Mannheim, Germany), $1 \mathrm{mg} / \mathrm{ml}$ collagenase (Sigma- 
Aldrich), and $100 \mu \mathrm{g} / \mathrm{ml}$ DNase I (Boehringer Mannheim) in RPMI for 1 hour at $37^{\circ} \mathrm{C}$. The cells were filtered through a nylon mesh, washed three times in RPMI plus 10\% FCS (Trace Bioscientific, Sydney, Australia), and stained for flow cytometric analysis. Nonadherent synovial cells were isolated following overnight incubation at $37^{\circ} \mathrm{C}$. Joint-exudate cells were collected by flushing of joints with $100 \mu \mathrm{l}$ of $10 \mathrm{mM}$ EDTA in PBS, cytocentrifugation of the synovial fluid, and staining with Diff-Quik reagents (Lab Aids, Narrabeen, Australia). Peritoneal exudate cells were obtained by peritoneal lavage with $5 \mathrm{ml}$ of RPMI plus 10\% FCS.

Flow cytometry. Synovial and LN cells were stained for flow cytometric analysis using FITC- and phycoerythrinconjugated antibodies to CD8 $\alpha$ (clone CT-CD8a), and phycoerythrin-conjugated antibodies to $\mathrm{CD} 11 \mathrm{~b}$ (clone $\mathrm{M} 1 / 70$ ) and CD4 (clone CT-CD4) (all from Caltag Laboratories Inc.). Biotinylated antibodies to CD45.2 (clone 104), GR-1 (clone RB6-8C5), CD25 (clone 7D4), CD44 (clone IM7), and CD69 (clone H1.2F3) were obtained from BD Pharmingen (San Diego, California, USA). Nonspecific binding of antibodies was blocked by preincubation of cells with an antibody to CD16 and CD32 (clone 2.4G2), and biotinylated antibodies were detected using streptavidin-TriColor (Caltag Laboratories Inc.). Cells were analyzed on a FACScan flow cytometer using CellQuest software (Becton Dickinson Immunocytometry Systems, San Jose, California, USA). Determination of $\beta$-gal activity by flow cytometry was carried out using the substrate fluorescein-D-galactopyranoside (FDG; Sigma-Aldrich), as described previously (23). Flow cytometric sorting experiments were performed using a FACSVantage SE flow cytometer (Becton Dickinson Immunocytometry Systems).

$T$ cell proliferation. Popliteal and axillary LN cells were cultured at a concentration of $2 \times 10^{5}$ cells per well in $200 \mu \mathrm{l} \mathrm{RPMI}$, supplemented with $1 \mathrm{mM}$ sodium pyruvate, $0.1 \mathrm{mM}$ nonessential amino acids (GIBCO BRL; Life Technologies Inc., Melbourne, Australia), and 10\% FCS. Cells were stimulated with $0-10 \mu \mathrm{g} / \mathrm{ml} \mathrm{mBSA}$ for 72 hours and were pulsed with $1 \mu \mathrm{Ci}$ tritiated thymidine $\left({ }^{3} \mathrm{H}-\mathrm{TdR}\right)$ for the final 8 hours. Thymidine incorporation was determined on a microplate scintillation counter (Canberra Packard, Melbourne, Australia) as a measure of $\mathrm{T}$ cell proliferation. Splenic $\mathrm{CD} 4^{+} \mathrm{T}$ cells were purified by labeling of the cells with a FITC-conjugated anti-CD4 antibody (clone GK1.5; Pharmingen, San Diego, California, USA) followed by antiFITC-labeled magnetic beads (Miltenyi Biotec Inc., Auburn, California, USA). $\mathrm{CD}^{+} \mathrm{T}$ cells were enriched to greater than $90 \%$ purity by autoMACS magnetic separation, according to the manufacturer's instructions (Miltenyi Biotec Inc.). Cells were cultured as described above in the presence of plate-bound anti$\mathrm{CD} 3$ antibodies, and proliferation was measured by ${ }^{3} \mathrm{H}-\mathrm{TdR}$ incorporation.

Statistical analysis. Data are expressed as mean \pm SEM. Differences in histological scores were analyzed using the Mann-Whitney two-sample rank test. Student's $t$ test was used to analyze the results of $\mathrm{T}$ cell proliferation assays. $P$ values less than 0.05 were considered significant.

\section{Results}

Detection of SOCS-1 in the inflamed synovium following induction of acute arthritis. To determine whether SOCS-1 is expressed in the synovium following the induction of acute arthritis, C57BL/6 mice were injected intra-articularly with mBSA, followed by three daily subcutaneous injections of IL-1. Mice were sacrificed on day 7, and knee joints were processed for histology. Knee joint sections were stained with goat polyclonal antibodies specific for SOCS-1, or with goat polyclonal antibodies of irrelevant specificity as a negative control.

Staining of sections with antibodies specific for SOCS-1 revealed expression of SOCS-1 protein within the synovium. The most prominent SOCS- 1 staining was in granulomas that had developed within the inflamed synovium by day 7 following mBSA injection (Figure 1, a and c). Staining was specific for SOCS-1, since no staining was detected in serial sections stained with isotype-control antibodies (Figure 1b). SOCS-1 expression was also detected in synovial cells found within pannus tissue, directly adjacent to bone (Figure 1d). No staining was detected within granulomas or pannus of knee joints of SOCS-1 $1^{-1-}$ IFN- $\gamma^{-/}$mice following induction of acute arthritis (Figure 1, $\mathrm{f}$ and $\mathrm{g}$ ).

IFN- $\gamma$ is not required for development of acute arthritis. Because the absence of SOCS-1 is associated with IFN- $\gamma$-dependent perinatal lethality, SOCS-1 $1^{-/-}$mice were maintained on an $I F N-\gamma^{/-}$background (20). It was therefore important to define the contribution of IFN- $\gamma$ to the development of acute arthritis in our model. WT or $I F N-\gamma^{/-}$mice, on a C57BL/6 background, were injected with mBSA in the knee joint and received three daily injections of IL- $1 \beta$ subcutaneously (mBSA/IL-1-induced arthritis). Arthritis was assessed on day 7 following mBSA injection. No difference in the incidence or overall severity of disease was seen between WT and $I F N-\gamma^{-/}$mice. Histological scores of individual features of arthritis in $I F N-\gamma^{/-}$ mice also did not differ significantly from those of WT mice (Figure 2). In addition, administration of neutralizing mAb's specific for IFN- $\gamma$ over the course of disease induction did not have any significant effect on the overall severity of disease, or on the severity of any of the five individual features (data not shown). IFN- $\gamma$ therefore does not play a significant role in the development of acute mBSA/IL-1-induced arthritis. All mice used in subsequent experiments were backcrossed to an $I F N-\gamma^{/-}$background, allowing for a direct comparison between SOCS-1 $1^{+/+} I_{F N-\gamma^{/-}}$and SOCS $-1^{-/-}$IFN- $\gamma^{-/}$mice.

Exacerbated synovial inflammation in the absence of SOCS-1. To assess the role of SOCS-1 in regulating the inflammatory and destructive features of acute arthritis, we compared the severity of disease in SOCS-1/++ $I F N-\gamma^{\prime-}$ and SOCS-1-/- IFN- $\gamma^{\prime-}$ mice. Knee joints were processed for histology on day 7 following mBSA 

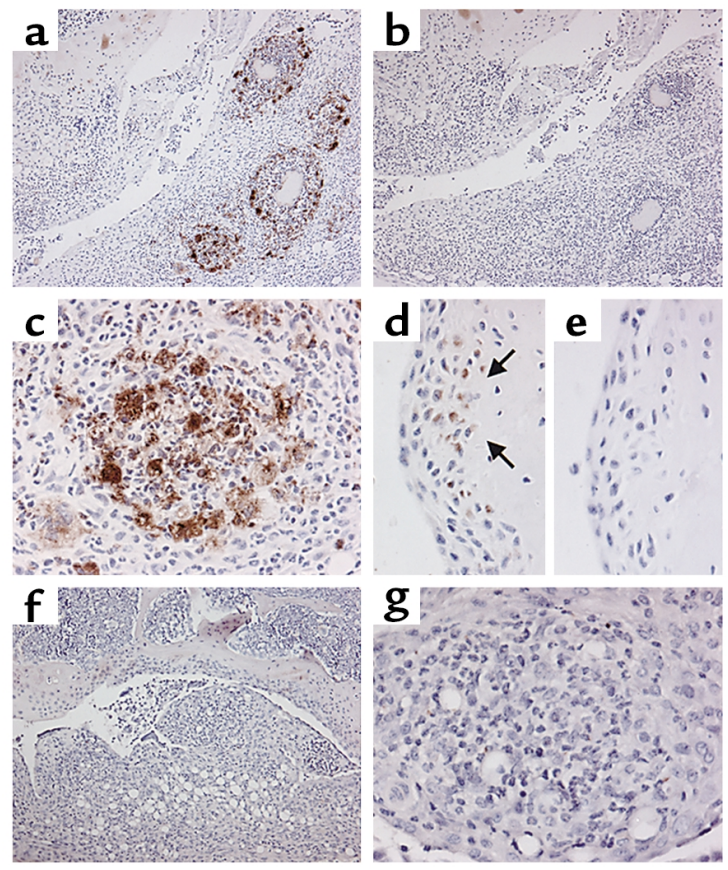

injection, and sections were graded by an investigator blinded to the treatment groups.

Joint pathology was seen in neither SOCS $-1^{+/+} \mathrm{IFN}_{-\gamma^{-/}}$ mice nor SOCS-1-/ IFN- $\gamma^{-/}$mice in the absence of mBSA injection (Figure 3 , $a$ and $b$ ). The knee joints of naive mice up to 12 weeks of age were examined and found to show no inflammatory features. In contrast, monoarticular arthritis was inducible in both SOCS-1+/+ IFN- $\gamma^{/-}$ and SOCS-1-1- IFN- $\gamma^{/-}$mice, and disease severity was exacerbated in the absence of SOCS-1 (Figure 3, c-f). The overall disease score, as measured by the sum of individual histological scores for the five features of arthritis examined, was significantly increased in SOCS $-1^{-/-}$mice $\left(16.9 \pm 1.3\right.$ for SOCS $-1^{-/-} I F N-\gamma^{-/}$mice compared with $10.2 \pm 0.6$ for SOCS-1 $1^{+/+} I F N-\gamma^{1-}$ mice, $P<0.005)$. Furthermore, the histological scores of each of the five features of arthritis were significantly increased in the absence of SOCS-1 (Figure 4a), indicating that both inflammatory features (joint-space exudate and synovitis) and destructive features (pannus formation and cartilage and bone destruction) were affected. To assess the role of SOCS- 1 in disease resolution, mice were also sacrificed on day 14 following mBSA injection (Figure $4 \mathrm{~b}$ ). At this time, arthritis was almost completely resolved in SOCS-1 $1^{+/+} I F N-\gamma^{/-}$mice. Residual arthritis was still more severe in the absence of SOCS-1, although the histological scores for each of the five arthritis features were reduced. SOCS- 1 can therefore regulate the extent of the inflammatory response, but disease resolution still proceeds in its absence.

Accumulation of myeloid cells in the synovium of SOCS-1-1$I F N-\gamma^{\prime-}$ mice during acute artbritis. To characterize the inflammatory cell infiltrate in synovium, we prepared a single-cell suspension of synovial tissue by enzymatic digestion and stained the cells for flow

\section{Figure 1}

Immunohistochemical staining for SOCS-1 during acute inflammatory arthritis. Acute inflammatory arthritis was induced by intraarticular injection of $200 \mu \mathrm{g}$ mBSA into the knee joint followed by three daily subcutaneous injections of $250 \mathrm{ng}$ IL-1 $\beta$. Mice were sacrificed on day 7 after mBSA injection. Paraffin-embedded joint sections were dewaxed and stained with purified goat polyclonal antibodies specific for SOCS-1 or goat polyclonal antibodies of irrelevant specificity. (a and $\mathbf{b}$ ) Inflamed WT synovium, stained with antibodies to SOCS- $1(\times 100, \mathbf{a})$ or with an isotype control $(\times 100$, b). (c) Inflammatory synovial granuloma from a WT mouse, stained with antibodies to SOCS-1 ( $\times 400)$. (d and e) Synovial pannus from a WT mouse, stained with antibodies to SOCS $-1(\times 400, \mathbf{d})$ or with an isotype control $(\times 400, \mathbf{e})$. Arrows in $\mathbf{d}$ indicate SOCS- 1 positive synovial cells at the cartilage-pannus junction. (f) SOCS-1-/-IFN- $\gamma^{-1}$ synovium stained with antibodies to SOCS-1 $(\times 100)$. $(\mathbf{g})$ Inflamed synovial granuloma from a SOCS-1-/- IFN- $\gamma^{-/}$mouse, stained with antibodies to SOCS-1 $(\times 400)$.

cytometry. CD45 was used as a marker of hematopoietic cells. The percentage of hematopoietic cells present in the synovium was increased from $38 \%$ in SOCS $-1^{+/+}{I F N-\gamma^{/-}}^{/}$mice to $66 \%$ in SOCS-1 $1^{-/-}{I F N-\gamma^{-/}}^{/-}$ mice on day 7 following mBSA injection (Figure 5a). $\mathrm{CD} 45^{+}$cells were further characterized by staining with antibodies specific for CD11b, which stains both monocyte/macrophages and granulocytes, as well as for GR-1, which is specifically expressed by neutrophils. The percentage of CD $11 \mathrm{~b}^{+} \mathrm{GR}-1^{-}$cells, which were defined as cells of the monocyte/ macrophage lineage, increased from $43 \%$ of cells in the synovium of SOCS-1//+ $I F N-\gamma^{-/}$mice to $51 \%$ of cells in SOCS $-1^{-/-} I F N-\gamma^{1-}$ mice. Similarly, the percentage of neutrophils, as defined by expression of both CD11b and GR-1 among the CD45-gated population of synovial cells, was increased from $35 \%$ in the SOCS-1 $1^{+/+}$ $I F N-\gamma^{/-}$mice to $40 \%$ in SOCS $-1^{-/-} I F N-\gamma^{/-}$mice.

Expression of CD4 or CD8 was not detected on freshly isolated synovial tissue cells. Expression of these molecules, however, is known to be sensitive to dispase

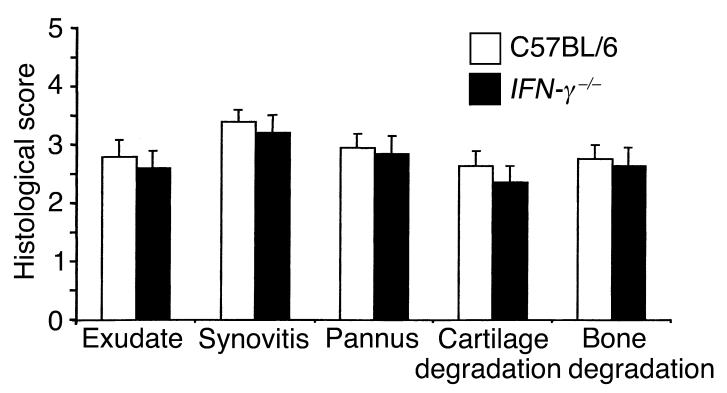

\section{Figure 2}

Histological assessment of acute inflammatory arthritis in C57BL/6 and $I F N-\gamma^{-/}$mice. Acute inflammatory arthritis was induced by intraarticular injection of $200 \mu \mathrm{g}$ mBSA into the knee joint followed by three daily subcutaneous injections of $250 \mathrm{ng}$ IL-1 $\beta$. Mice were sacrificed on day 7 following mBSA injection. H\&E-stained joint sections were graded for five features of inflammatory arthritis, each on a scale of 0 (normal) to 5 (severe). Results shown are the mean + SEM of 16 knee joints and are representative of three independent experiments. 


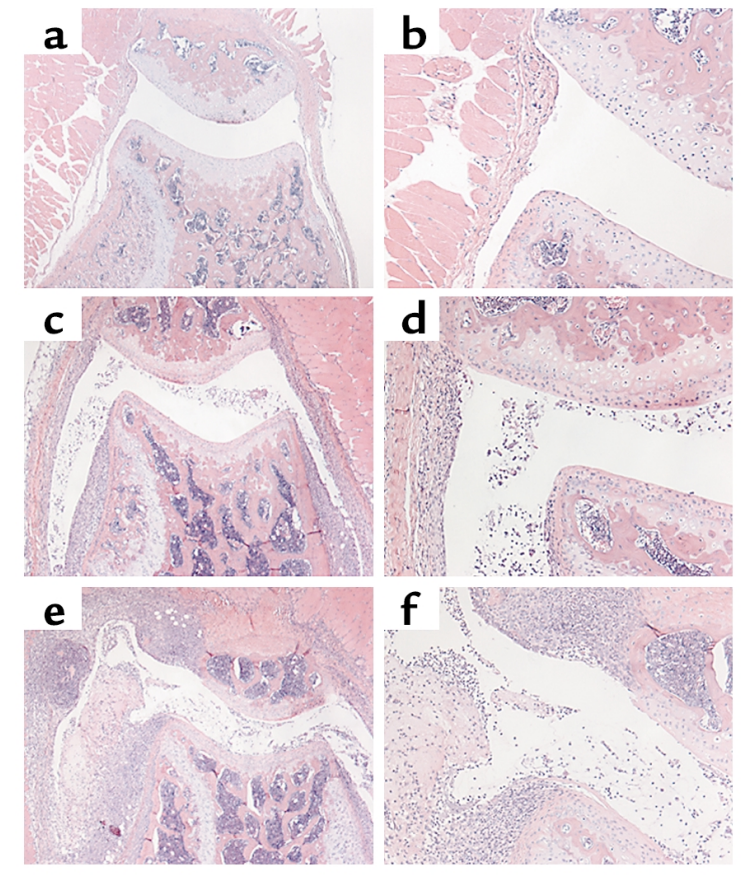

Figure 3

Histopathology of joints from SOCS $-1^{+/+} \mid F N-\gamma^{/-}$and SOCS-1-/IFN- $\gamma^{-1-}$ mice. Acute inflammatory arthritis was induced by intraarticular injection of $200 \mu \mathrm{g}$ mBSA into the knee joint followed by three daily subcutaneous injections of $20 \mathrm{ng}$ IL-1 $\beta$. Mice were sacrificed on day 7 , knee joints were fixed in $10 \%$ formalin, and H\&Estained frontal sections were prepared. Knee joints were taken from nonarthritic SOCS-1-/- IFN- $\gamma^{-/-}$mice ( $\mathbf{a}$ and $\mathbf{b}$ ), arthritic SOCS $-1^{+/+} \mid F N-\gamma^{/-}$mice (c and d), and arthritic SOCS-1 $1^{-/-} \mid F N-\gamma^{\prime-}$ mice (e and $\mathbf{f}$ ). Magnification: $\mathbf{a}, \mathbf{c}$, and $\mathbf{e}, \times 40 ; \mathbf{b}, \mathbf{d}$, and $\mathbf{f}, \times 100$.

digestion, so it is likely that these markers were cleaved from the cells during isolation from the synovium. Following overnight incubation of synovial cells at $37^{\circ} \mathrm{C}, \mathrm{CD} 4^{+}$cells were detected in the nonadherent cell population. The percentage of $\mathrm{CD}^{+}$synovial cells increased from $2.3 \%$ in SOCS-1 $1^{+/} \mathrm{IFN}^{-\gamma^{-/}}$mice to $4.0 \%$ in SOCS-1/-- IFN- $\gamma^{-/}$mice (Figure $5 \mathrm{~b}$ ). No $\mathrm{CD}^{+}$cells were detected in the synovium of either strain of mice. Joint-exudate cells were collected by joint lavage with $10 \mu \mathrm{M}$ EDTA in PBS, and cells were centrifuged onto slides for morphological analysis (Figure 5c). The cellular composition of exudate cells from SOCS $1^{+/+}{\mathrm{IFN}-\gamma^{/-}}^{/-}$mice comprised approximately $50 \%$ granulocytes and $30 \%$ macrophages. In contrast, the joint exudate of SOCS-1 $1^{-/} I F N-\gamma^{/-}$mice comprised approximately $70 \%$ macrophages and $22 \%$ granulocytes. The absence of SOCS-1 therefore

\section{Figure 4}

Histological assessment of acute inflammatory arthritis in SOCS-1+/+ IFN- $\gamma^{-/}$and SOCS-1-/- IFN- $\gamma^{-/-}$mice. Joint sections were graded for five features of inflammatory arthritis, each on a scale of 0 (normal) to 5 (severe). Mice were sacrificed on day 7 (a) or day 14 (b) after mBSA injection. Results shown are the mean + SEM of ten knee joints and are representative of three independent experiments. ${ }^{*} P<0.005$, $\dagger P<0.01$ by Mann-Whitney test. results in an accumulation of macrophages in the joint space during the development of arthritis.

SOCS-1 promoter activity in the synovial compartment during arthritis. The targeted disruption of the SOCS-1 gene introduced a $\beta$-gal reporter gene into the SOCS-1 locus (19). Expression of $\beta$-gal activity could therefore be used to determine the identity of cells expressing SOCS-1 promoter activity following induction of acute arthritis. Synovial cells were isolated by enzymatic digestion of the synovium from SOCS-1 $1^{-/-} I F N-\gamma^{-1}$ mice on day 7 following arthritis induction. Cells were exposed to the $\beta$-gal substrate FDG, which yields a fluorescent product when cleaved; they were then stained with phenotypic markers and analyzed by flow cytometry.

Staining with antibodies specific for CD 45 and CD11b was used to identify myeloid $\left(\mathrm{CD} 45^{+} \mathrm{CD} 11 \mathrm{~b}^{+}\right)$, lymphoid $\left(\mathrm{CD} 45^{+} \mathrm{CD} 11 \mathrm{~b}^{-}\right)$, and stromal $\left(\mathrm{CD} 45^{-} \mathrm{CD} 11 \mathrm{~b}^{-}\right)$cells present in the synovium. Although the process of enzymic digestion of the synovial tissue cleaved some phenotypic markers from the cells, such as $\mathrm{CD} 4$ for $\mathrm{T}$ cells and VCAM- 1 for fibroblasts, light-scatter properties of each subpopulation were consistent with those expected for each cell type (Figure 6a). CD $45^{+} \mathrm{CD} 11 \mathrm{~b}^{+}$cells had high forward and side scatter, consistent with this population being predominantly myeloid cells. In contrast, $\mathrm{CD}^{2} 5^{+} \mathrm{CD} 11 \mathrm{~b}^{-}$cells expressed a low forward- and sidescatter profile, consistent with the cells being predominantly lymphocytes. CD45- CD11 b- cells were a heterogeneous population but were predominantly large, granular cells as determined by light scatter, consistent with these cells being predominantly synovial fibroblasts. In addition, this population of cells assumed a typical fibroblast morphology when allowed to adhere in culture for 4 hours (data not shown). Gating on each subpopulation revealed that significant proportions of all three populations expressed $\beta$-gal activity. The percentage of
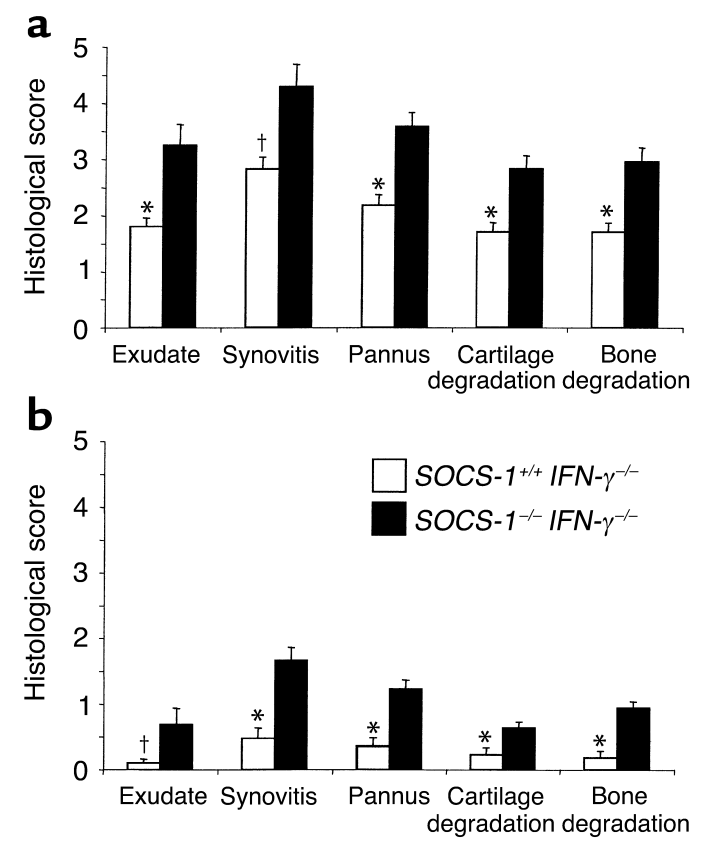
a
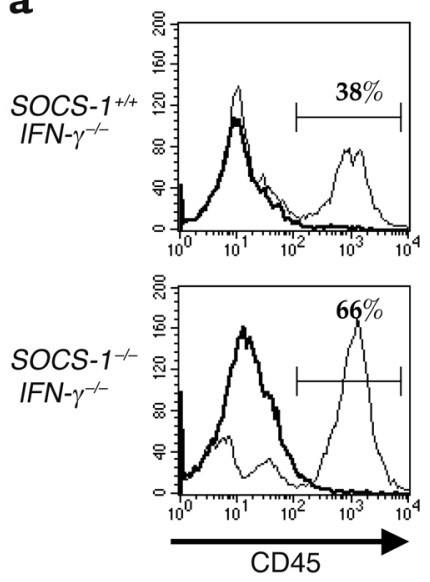

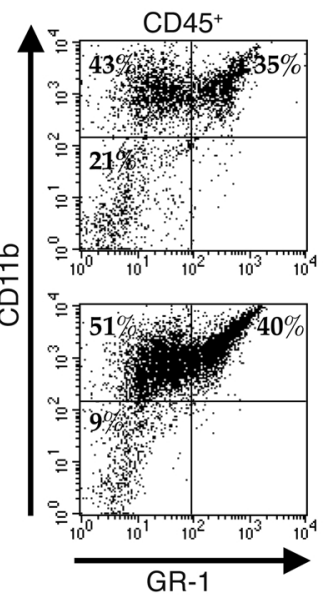

b
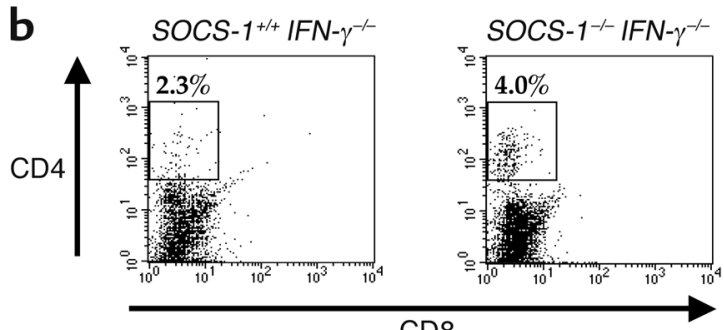

C

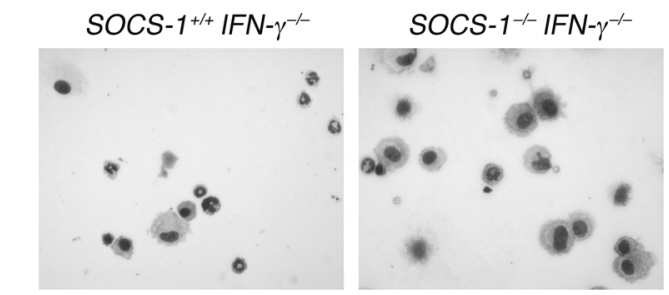

\section{Figure 5}

Phenotype of cells accumulating in the synovium of SOCS-1-/- IFN- $\gamma^{-1-}$ mice during acute arthritis. Synovium was dissected from the knee joints of SOCS $1^{+/+} I F N-\gamma^{/-}$and SOCS-1-/- IFN- $\gamma^{-/}$mice on day 7 after arthritis induction, and synovial cells were isolated by digestion with dispase and collagenase. (a) Increased percentage of myeloid cells in the synovium of SOCS-1-/-IFN- $\gamma^{-1}$ mice during acute arthritis. Synovial cells were stained for expression of CD45, and analysis of CD11b and GR-1 expression was performed on CD45 cells. Results shown are representative of three independent experiments. (b) Flow cytometric analysis of nonadherent synovial cells for expression of CD4 and CD8. Nonadherent synovial cells were isolated following overnight incubation. (c) Cytocentrifuge preparations of joint exudate cells, isolated on day 7 following arthritis induction. $\times 400$.

$\beta$-gal ${ }^{+}$cells was $45 \%$ for $\mathrm{CD} 45^{+} \mathrm{CD} 11 \mathrm{~b}^{+}$myeloid cells, $70 \%$ for $\mathrm{CD}^{+} 5^{+} \mathrm{CD} 11 \mathrm{~b}^{-}$lymphoid cells, and $60 \%$ for $\mathrm{CD} 45^{-}$ synovial cells. Synovial cells isolated from SOCS-1 ${ }^{++}$ $I F N-\gamma^{\prime}$ mice, which do not contain the reporter gene, did not show any significant $\beta$-gal activity (data not shown).

Since there appeared to be a distinct subpopulation of $\mathrm{CD}_{11} \mathrm{~b}^{+}$cells that were positive for $\beta$-gal activity, CD $11 \mathrm{~b}^{+}$ synovial cells were sorted by flow cytometry, based on $\beta$-gal activity, in order to compare the morphology of cells that did or did not express $\beta$-gal. Over $90 \%$ of CD $11 b^{+}$ $\beta$-gal ${ }^{-}$cells were neutrophils, while the remaining cells displayed a monocyte-like phenotype (Figure 6b). In contrast, $\mathrm{CD} 11 \mathrm{~b}^{+} \beta$-gal ${ }^{+}$cells were virtually all activated macrophages, being larger cells with a distinctive foamy cytoplasm and containing large vacuoles. CD $11 \mathrm{~b}^{+}$cells that expressed $\beta$-gal activity were also larger and more granular by forward/side scatter analysis than cells negative for $\beta$-gal, consistent with an activated phenotype.

Reporter gene expression in synovial macrophages following induction of inflammatory arthritis was compared with expression in macrophages isolated from a noninflamed site. Resident peritoneal cells were isolated from naive SOCS-1 $1^{-/-} I F N-\gamma^{/ /}$mice and stained for CD $11 b$ and $\beta$-gal activity (Figure 6c). No $\beta$-gal activity was detected in $\mathrm{CD} 11 \mathrm{~b}^{+}$resident peritoneal macrophages in the absence of an inflammatory stimulus, suggesting that reporter gene expression seen in synovial macrophages was induced in response to inflammation in the synovium. Induction of inflammatory arthritis therefore induced SOCS-1 promoter activity in a number of different cell lineages present in the synovium, including macrophages, lymphocytes, and fibroblasts. Induction of SOCS-1 promoter activity was not ubiquitous, however, since it was not detected in granulocytes isolated from the synovium. Lymphadenopathy in draining LNs. Since the mBSA/IL-1 model of acute arthritis is a CD4-dependent response, we examined the cellular phenotype of cells within LNs on day 7 following arthritis induction. The LNs analyzed were the popliteal LNs, which are the major draining nodes for the knees, and axillary LNs, which do not drain the knees and were therefore analyzed as negative controls. Because of the small size of individual popliteal LNs, results shown represent average LN size calculated from pooled LN cells.

In three independent experiments, there were up to fourfold more cells in the popliteal LNs of SOCS-1 $1^{-/-}$ $I F N-\gamma^{\prime-}$ mice compared with similar LNs taken from SOCS-1 $1^{+/+} I F N-\gamma^{-/}$mice on day 7 following mBSA injection (Table 1). There was also a slight increase in the total number of cells present in the nondraining axillary LNs from SOCS $1^{-/-} \mathrm{IFN}-\gamma^{-/}$mice, compared with SOCS-1 $1^{+/+}$ $I F N-\gamma^{\prime-}$ mice, following induction of arthritis. The lymphoid organs of unchallenged SOCS-1-/-IFN- $\gamma^{/-}$mice, however, show a similar increase in the total number of cells when compared with those of SOCS-1+/+ IFN- $\gamma^{/-}$ mice, which is predominantly due to an expansion of $\mathrm{CD}^{+} \mathrm{T}$ cells (24); and the increased number of cells seen in axillary LNs following induction of arthritis was of similar magnitude. In contrast, the increased cellularity seen in draining LNs following the induction of acute arthritis was disproportionately greater. Induction of acute arthritis in the absence of SOCS-1 therefore induces lymphadenopathy in the draining LNs.

SOCS-1-/ T cells are byperproliferative in vitro. One possible reason for the increase in cellularity in draining 

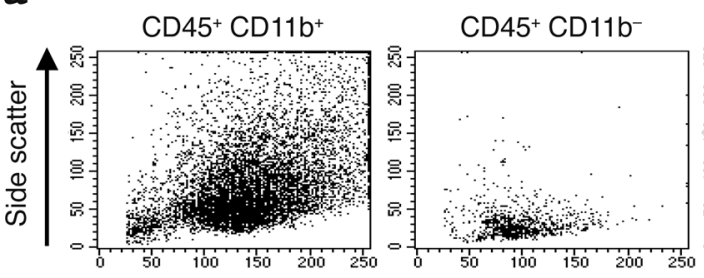

Forward scatter
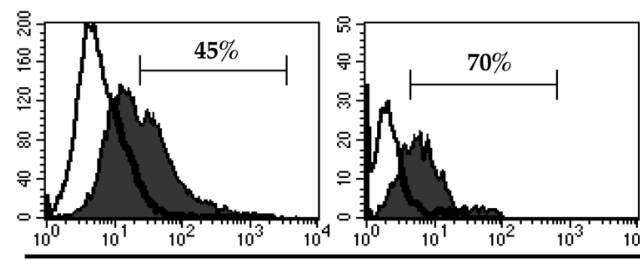

$\beta$-gal b
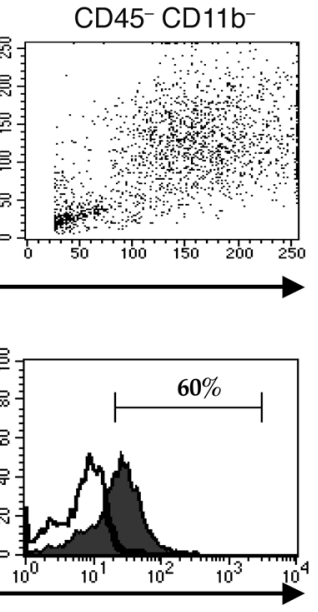

C
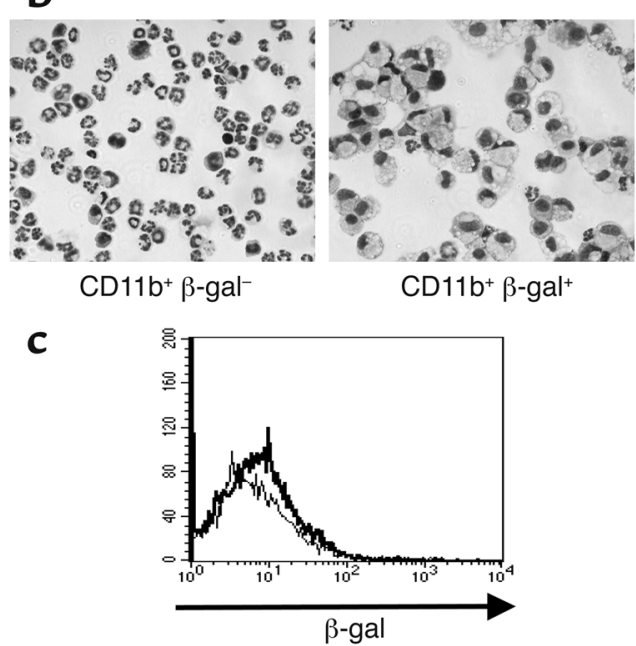

Figure 6

Expression of $\beta$-gal reporter gene activity in synovial tissue cells from SOCS-1 ${ }^{-1-}$ IFN- $\gamma^{-1}$ mice on day 7 of acute arthritis. Synovial tissue cells were isolated by collagenase and dispase digestion of dissected synovium and incubated with FDG for 4 hours. Cells were then stained for expression of phenotypic markers. (a) Cells were stained with CD45 and CD11b, and gated populations were analyzed by light scatter (top row) and $\beta$-gal reporter gene activity (bottom row). Cells were incubated in the presence (thin histogram) or absence (bold histogram) of FDG for 4 hours, followed by staining with phenotypic markers. (b) Cytocentrifuge preparations of CD11 $b^{+}$synovial cells, sorted by flow cytometry on the basis of $\beta$-gal activity (Diff-Quik stain; magnification $\times 400$ ). Results shown are representative of three independent experiments. (c) Lack of reporter gene expression in resident peritoneal macrophages. Resident peritoneal cells from naive SOCS-1-/- IFN- $\gamma^{-1-}$ mice were stained for $\mathrm{CD} 11 \mathrm{~b}^{+}$expression and $\beta$-gal activity. Cells were incubated in the presence (thin histogram) or absence (bold histogram) of FDG for 4 hours. Results shown are gated for CD11 $\mathrm{b}^{+}$cells.

LNs of SOCS-1-/-IFN- $\gamma^{-/}$mice is that T cells within the nodes are hyperresponsive to stimulatory cytokines, such as IL-2, that are produced following intra-articular injection of antigen. In order to test this, LN cells from arthritic mice were cultured in vitro in the presence of mBSA, and cellular proliferation was assessed by ${ }^{3} \mathrm{H}-\mathrm{TdR}$ incorporation. While popliteal or axillary LN cells from SOCS-1+/+ IFN- $\gamma^{/-}$mice showed no significant proliferation in response to up to $10 \mu \mathrm{g} / \mathrm{ml}$ mBSA, popliteal LNs from SOCS-1 $1^{-/} I F N-\gamma^{-1}$ mice strongly proliferated in response to mBSA (Figure 7a). In addition, popliteal LN cells from SOCS-1 $1^{-/-} I F N-\gamma^{-1}$ mice also showed significant proliferation in the absence of any exogenous antigen. Overall, proliferation of popliteal LN cells from SOCS-1 $1^{-/-} I F N-\gamma^{\prime-}$ mice was increased 10- to 15-fold compared with cells from SOCS-1 ${ }^{+/+} I F N-\gamma^{/-}$mice. Cells from SOCS-1 $1^{-/} \mathrm{IFN}_{-} \gamma^{/-}$ axillary LNs, which do not drain the knee, did not proliferate in response to $\mathrm{mBSA}$ or to media alone. Similarly, there was no significant difference in the proliferation of popliteal LN cells of naive SOCS-1+/+ $I F N-\gamma^{/-}$and SOCS-1-/-IFN- $\gamma^{/-}$mice (data not shown).

Purified splenic $\mathrm{CD}^{+}{ }^{+} \mathrm{T}$ cells from naive SOCS $-1^{+/+}$ $I F N-\gamma^{\prime-}$ and SOCS-1 $1^{-/-} I F N-\gamma^{\prime-}$ mice were cultured with anti-CD3 to evaluate whether the absence of SOCS-1 causes hyperproliferation following $\mathrm{T}$ cell receptor signaling (Figure $7 \mathrm{~b}$ ). $T$ cells were isolated to greater than $90 \%$ purity by magnetic-bead separation and cultured with increasing concentrations of immobilized antiCD3. CD $4^{+} \mathrm{T}$ cells from SOCS $-1^{-/-} I F N-\gamma^{-/}$mice showed an approximately fivefold increased proliferative response following stimulation with anti-CD3. $\mathrm{CD}^{+}$ $\mathrm{T}$ cells from SOCS-1/- IFN- $\gamma^{-/-}$mice also responded to a lower threshold of stimulation, compared with cells purified from spleens of SOCS-1+/+ IFN- $\gamma^{-/}$mice. SOCS- 1 is therefore involved in regulation of the T cell proliferative response following activation.

Expression of SOCS-1 promoter activity in activated $T$ cells. LN cells were harvested from SOCS-1-/ IFN- $\gamma^{/-}$mice on day 7 following induction of arthritis and were stained

Table 1

Increased cellularity of draining LNs in SOCS-1-/- IFN- $\gamma^{-/}$mice following arthritis induction ${ }^{\mathrm{A}}$

\begin{tabular}{|c|c|c|c|c|c|c|}
\hline \multirow{3}{*}{$\begin{array}{l}\text { Experiment } \\
S O C S-1^{B}\end{array}$} & \multicolumn{6}{|c|}{ Total cells per LN } \\
\hline & \multicolumn{2}{|c|}{1} & \multicolumn{2}{|c|}{2} & \multicolumn{2}{|c|}{3} \\
\hline & $+/+$ & $-/-$ & $+/+$ & $-/-$ & $+/+$ & $-/-$ \\
\hline Popliteal & $5 \times 10^{5}$ & $2.3 \times 10^{6}$ & $3 \times 10^{5}$ & $7 \times 10^{5}$ & $5 \times 10^{5}$ & $2.0 \times 10$ \\
\hline Axillary & $1.5 \times 10^{6}$ & $1.9 \times 10^{6}$ & $1.3 \times 10^{6}$ & $1.4 \times 10^{6}$ & $3.1 \times 10^{6}$ & $5.8 \times 10$ \\
\hline
\end{tabular}

ATotal cell numbers obtained from LN cells pooled from at least four mice on day 7 after mBSA injection. ${ }^{B}$ All mice were IFN- $\gamma^{\prime-}$. 


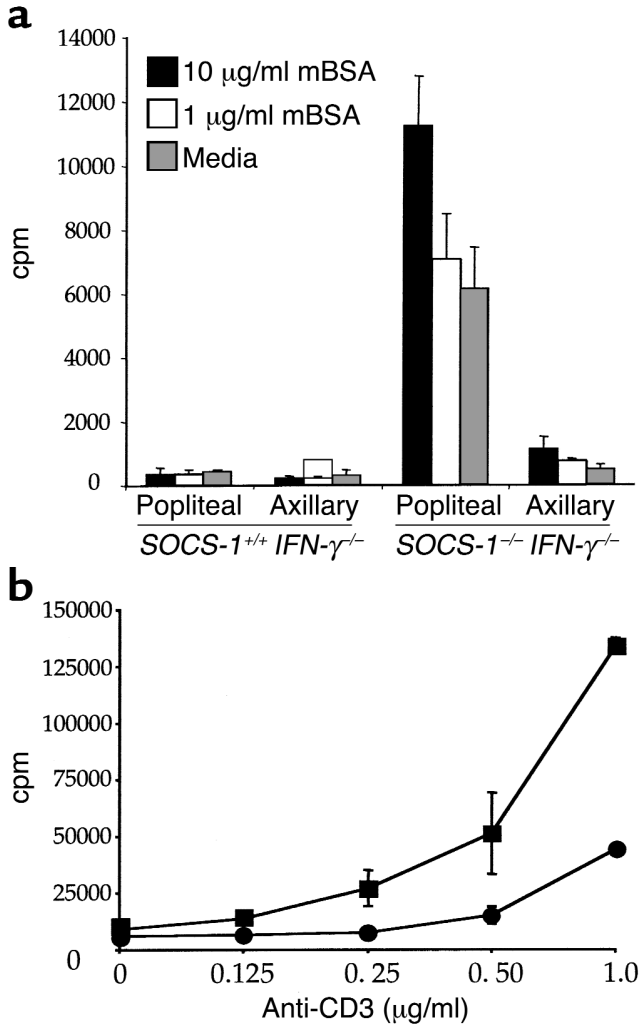

for $\beta$-gal activity. Expression of the reporter gene was correlated with the expression of the activation markers CD25, CD44, or CD69 on popliteal LN cells (Figure 8). Resting or naive $\mathrm{CD} 4^{+} \mathrm{T}$ cells that did not express activation markers were negative for $\beta$-gal activity. In contrast, the percentage of $\mathrm{CD}^{+} \mathrm{T}$ cells expressing $\beta$-gal activity was $21 \%, 26 \%$, and $27 \%$ for $\mathrm{CD} 25^{+}, \mathrm{CD} 44^{+}$, and $\mathrm{CD} 69^{+}$ cells, respectively, demonstrating that SOCS-1 promoter activity in the $\mathrm{CD}^{+} \mathrm{T}$ cell compartment was confined to cells with an activated or memory phenotype. Similar proportions of $\beta$-gal ${ }^{+}$cells were also seen in $\mathrm{CD}^{+} \mathrm{T}$ cells expressing $\mathrm{CD} 25$, CD44, or CD69, while $\mathrm{CD}^{+} \mathrm{T}$ cells that lacked expression of activation markers were negative for $\beta$-gal activity (data not shown). Comparable results were also obtained for $\mathrm{CD}^{+}$and $\mathrm{CD}^{+} \mathrm{T}$ cells isolated from axillary LNs, suggesting that SOCS-1 expression is a general feature of T cell activation.

\section{Discussion}

SOCS-1 was discovered through its ability to inhibit signaling of IL-6, via inhibition of STAT1 phosphorylation (9-11). Subsequent in vitro and in vivo experiments have

\section{Figure 8}

Expression of $\beta$-gal reporter gene activity in popliteal $\mathrm{LN} \mathrm{CD4} 4^{+} \mathrm{T}$ cells. Popliteal LN cells from SOCS $-1^{-/-} \mid F N-\gamma^{\prime-}$ mice were isolated on day 7 of acute arthritis and incubated with FDG for 4 hours. Cells were then stained for expression of CD4 and the activation markers CD25, CD44, and CD69. CD4 ${ }^{+} T$ cells were gated according to the absence (R1) or presence (R2) of activation markers, and $\beta$-gal activity of the gated populations of cells is shown.

\section{Figure 7}

Hyperresponsiveness of SOCS-1-deficient T cells in vitro. (a) Enhanced proliferation of draining LN cells from SOCS-1-/- IFN- $\gamma^{-1-}$ mice to mBSA. Popliteal and axillary LN cells were isolated from SOCS $-1^{+/+}$IFN- $\gamma^{-/-}$and SOCS-1-/- IFN- $\gamma^{-/}$mice and cultured in vitro for 3 days in the presence or absence of mBSA. (b) Hyperresponsiveness of purified $C D 4^{+} T$ cells to anti-CD3. Splenic $C D 4^{+} T$ cells were purified from nonarthritic SOCS $-1^{+/+} I F N-\gamma^{-/}$mice (circles) and SOCS $-1^{-/-}$IFN $-\gamma^{-1}$ mice (squares) and cultured with increasing concentrations of immobilized anti-CD3 antibody. T cell proliferation was assessed after 3 days in culture by incorporation of ${ }^{3} \mathrm{H}-\mathrm{TdR}$.

shown that SOCS-1 can inhibit the signal transduction pathways of multiple cytokine receptors, including those for IFN- $\gamma$, IL-2, IL-3, IL-5, and TNF- $\alpha$. Mice lacking SOCS-1 die at weaning, of an inflammatory syndrome characterized by fatty degeneration of the liver and mononuclear cell infiltration into numerous organs (19). This syndrome is dependent on hyperresponsiveness to IFN- $\gamma$ signaling, since mice doubly deficient in SOCS- 1 and IFN- $\gamma$ survive until adulthood (20). Longterm survival of SOCS-1 $1^{-/} I F N-\gamma^{/-}$mice, however, is impaired, due to the development of multiple inflammatory lesions in the skin, gut, and other organs, pneumonia, and polycystic kidneys (25), suggesting that, in vivo, SOCS-1 is responsible for negative regulation of other cytokines in addition to IFN- $\gamma$. Given that SOCS-1 is an important physiological regulator of cytokine signaling, mice lacking SOCS-1 may develop more severe inflammatory diseases. In vitro evidence suggests that SOCS-1 has the potential to regulate the development of inflammatory responses, through inhibition of proinflammatory cytokines such as IL-6 and GM-CSF, and T cell activation, via effects on cytokines such as IL-2 and
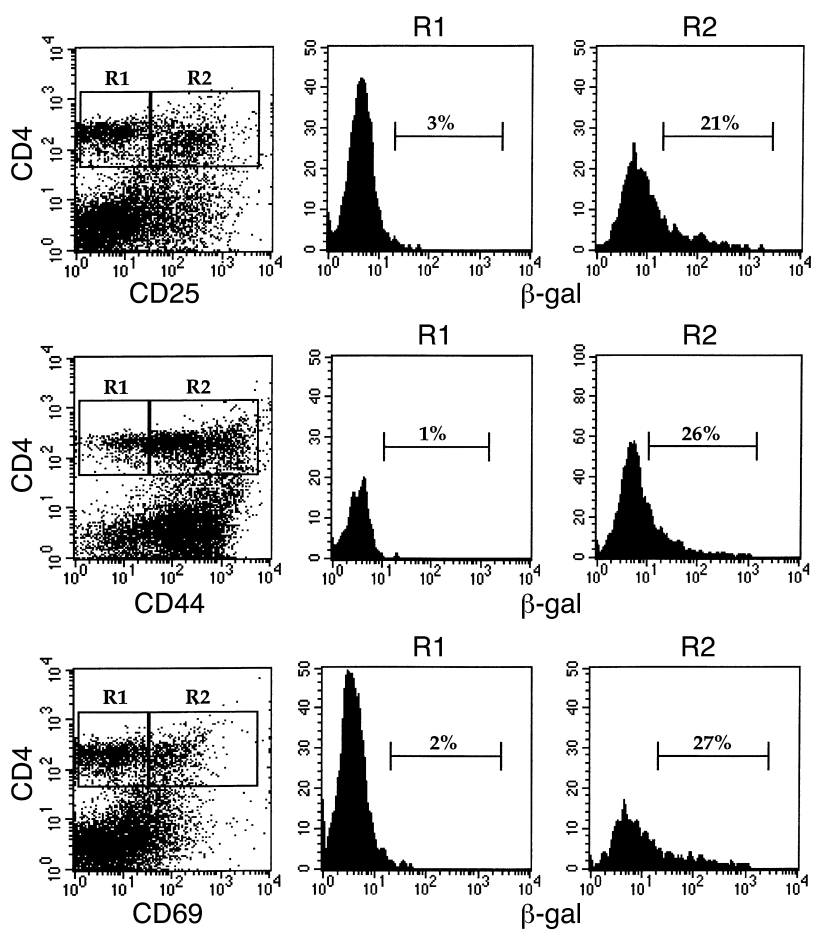
IL-4 (reviewed in ref. 8). We have used an acute inflammatory arthritis model to examine these issues.

The advantages of the mBSA/IL-1 model are the rapid and reproducible development of arthritis in the injected knee joint and the involvement of $\mathrm{CD}^{+} \mathrm{T}$ cells in the development of disease. We have previously shown that the development of disease is dependent on $\mathrm{CD}^{+} \mathrm{T}$ cells, while $\mathrm{CD}^{+} \mathrm{T}$ cells and $\mathrm{B}$ cells are not required (ref. 22; K.E. Lawlor and I.P. Wicks, unpublished observations). Although IL-1 drives the mBSA/IL-1 model of acute arthritis, SOCS-1 is not thought to be a regulator of IL-1 signaling. The exact mechanism by which IL-1 induces disease, however, is not known, but it is likely to involve the induction of other proinflammatory and immunostimulatory cytokines whose activity may be modulated by SOCS-1. Importantly, the development and severity of arthritis was not affected in the absence of IFN- $\gamma$; thus SOCS $-1^{-/-} I F N-\gamma^{-1}$ mice can be used in this model. This model of disease, however, is dependent on other cytokines such as GM-CSF, TNF- $\alpha$, and IL-6, since mice deficient in these cytokines have reduced arthritis (refs. 7, 26; P. Wong and I.P. Wicks, unpublished data). Since SOCS-1 has been shown to modulate signaling through these cytokines, we have examined the effects of a lack of SOCS-1 on the development of acute arthritis, both within the synovium and in the draining LNs.

Our results suggest that SOCS-1 is involved in regulating the course of arthritis in two distinct compartments. Firstly, the inflammatory response in the synovium was more severe in mice lacking SOCS-1. Secondly, SOCS-1 was also involved in regulating the extent of $\mathrm{T}$ cell proliferation and activation in the draining $\mathrm{LN}$. The major feature of arthritis seen in SOCS $1^{-/-} I F N-\gamma^{\prime-}$ mice was increased severity of synovial inflammation, characterized by greater infiltration of myeloid cells into the inflamed joint. In addition, destructive features of arthritis, such as pannus formation and cartilage and bone degradation, were also more severe in the absence of SOCS-1. Although some of these effects could be secondary to dysregulated cytokine production, SOCS-1 promoter activity was seen in synovial macrophages and fibroblasts, suggesting that increased severity of disease also involves an enhanced response of these cells to cytokine stimulation. SOCS-1 promoter activity, however, was not ubiquitous, as granulocytes isolated from the synovium of arthritic mice did not contain detectable $\beta$-gal activity. In addition, although sorted populations of $\mathrm{CD}_{11 \mathrm{~b}^{+} \beta \text {-gal }}{ }^{+}$cells were predominantly activated macrophages, approximately $10 \%$ of $\mathrm{CD}_{11} \mathrm{~b}^{+} \beta$-gal ${ }^{-}$cells were monocytes. This suggests that SOCS- 1 is expressed at a distinct stage in the macrophage differentiation and activation pathway and is likely to occur in response to cytokine stimulation. Detection of SOCS-1 promoter activity in macrophages is consistent with immunohistochemical detection of SOCS-1 within granulomas that formed in the inflamed synovium following induction of disease and within pannus tissue (Figure 1), since activated macrophages are present in both sites. Accumula- tion of macrophages in the joint exudate of mice lacking SOCS-1 following induction of arthritis is also consistent with a role of SOCS-1 in the negative regulation of macrophage differentiation and activation following induction of disease (Figure 5c).

Although we did not detect SOCS-1 promoter activity within granulocytes isolated from the inflamed synovium, we cannot rule out the possibility of a low level of SOCS-1 gene expression below the level of detection of the assays used. The level of expression, however, would be significantly lower than that in activated macrophages, lymphocytes, or fibroblasts. Detection of SOCS- 1 promoter activity in synovial fibroblasts is consistent with a previous report demonstrating that SOCS $-1^{-/-}$synovial fibroblasts are hyperproliferative to stimulation with IL-6 (27), a proinflammatory cytokine that is produced at high levels in inflamed synovium. Production of MMPs, which are primary mediators of cartilage and bone destruction, is also induced by IL-6. SOCS-1 is therefore likely to be important in negative regulation of both synovial fibroblast proliferation and tissue remodeling during the onset of arthritis.

The most striking lymphoid defects seen in the absence of SOCS-1 were a profound lymphadenopathy in the draining LNs and T cell hyperresponsiveness in vitro. Popliteal LN cells from SOCS $-1^{-/-} I F N-\gamma^{--}$mice at the peak of the arthritic response spontaneously proliferated in the absence of exogenous stimulation, and stimulation with antigen increased proliferation further. Since axillary LNs did not show this response, it is likely that the popliteal LN cells were hyperresponsive to stimulatory signals, such as IL-2, received in vivo following activation. Similarly, purified $\mathrm{CD}^{+}$ $\mathrm{T}$ cells from untreated SOCS $-1^{-/-} I F N-\gamma^{/-}$mice were hyperresponsive to stimulation with anti-CD3. There have been a number of reports implicating SOCS- 1 in the negative regulation of IL-2 signaling and $\mathrm{T}$ cell homeostasis. SOCS-1 is induced in human peripheral blood T cells following stimulation with IL-2 and can inhibit STAT5 phosphorylation by binding to JAK1 (28). We now show that SOCS-1 can regulate the magnitude of the $T$ cell response to an exogenous antigen in vivo and, following engagement of the $T$ cell receptor, by anti-DC3 stimulation in vitro. While it is likely that the increased proliferation is due to a dysregulated response to IL- 2 induced by $\mathrm{T}$ cell receptor engagement, in vitro reconstitution experiments have demonstrated a possible role for SOCS-1 in the inhibition of $\mathrm{CD} 3 \zeta$-mediated signaling (29), suggesting that signaling through the $\mathrm{T}$ cell receptor itself is also regulated by SOCS -1 . The finding that $\beta$-gal reporter gene activity was found in $\mathrm{CD}^{+}{ }^{+} \mathrm{T}$ cells expressing the activation markers CD25, CD44, and CD69, but not in naive $\mathrm{CD}^{+} \mathrm{T}$ cells, confirms that SOCS- 1 is transcribed following $\mathrm{T}$ cell activation.

Preliminary RNase protection analysis of mRNA isolated from the synovium of SOCS $-1^{+/+} I F N-\gamma^{/-}$and SOCS $-1^{-/-} I F N-\gamma^{/-}$mice following the induction of arthritis did not detect any significant difference in 
the amount of RNA coding for the inflammatory cytokines IL-1 $\alpha$, IL-1 $\beta$, IL- 6 , or IL-18. Although many other inflammatory mediators might be increased, and the extent of synovial inflammation is greater in mice lacking SOCS-1, this would suggest that exacerbated disease is mainly a result of hyperresponsiveness of cells to stimulation with cytokines, rather than overproduction of cytokines. This suggestion is consistent with the known activity of SOCS-1 in inhibiting intracellular signaling.

Although arthritis was more severe in SOCS-1 $1^{-/-} \mathrm{IFN}^{-\gamma^{-/}}$ mice, resolution of disease was occurring by day 14 , although the disease was still more severe than in SOCS $-1^{+/+} I F N-\gamma^{-/}$mice. Whether this was because disease resolution was slower in the absence of SOCS-1 or because of the increased peak severity of disease has yet to be resolved. Apart from SOCS-1, other members of the SOCS family may also be involved in regulating cytokine stimulation during arthritis induction. SOCS-3-specific mRNA, for example, was produced by synovial cells during arthritis, and adenovirusmediated transfer of SOCS-3 into the ankle joints of mice with CIA has been shown to reduce the incidence and severity of disease (30). Because SOCS-3-deficient mice die in utero, it is not possible to directly assess the course of arthritis in the absence of SOCS-3, although this could be done through the generation of conditional knockout mice lacking SOCS-3 in defined cellular compartments.

In summary, SOCS-1 is an important negative regulator of experimental arthritis and has multiple effects depending on cell lineage. In the synovium, it acts in both macrophages and fibroblasts to limit the extent of inflammation and joint destruction, while in the draining LNs, it is important in regulating the extent of $\mathrm{T}$ cell expansion following antigen stimulation. These pleiotropic effects can be attributed to the actions of SOCS-1 regulating multiple cytokine responses within different cell lineages. Agents designed to induce the expression of SOCS-1 may offer a new therapeutic strategy for the treatment of inflammatory arthritis.

\section{Acknowledgments}

This work was supported by the Reid Charitable Trusts and the Arthritis Foundation of Australia. P.J. Egan is an Industry Research Fellow and I.P. Wicks is a Clinical Practitioner Fellow of the National Health and Medical Research Council of Australia. We thank Ian Campbell for helpful discussion, Doug Hilton for critical review of the manuscript, Danielle Cooper for animal care, and Steven Mihajlovic and Ellen Tsui for histology.

\footnotetext{
1. Feldmann, M., Brennan, F.M., and Maini, R.N. 1996. Role of cytokines in rheumatoid arthritis. Annu. Rev. Immunol. 14:397-440.

2. Mori, L., Iselin, S., De Libero, G., and Lesslauer, W. 1996. Attenuation of collagen-induced arthritis in 55-kDa TNF receptor type 1 (TNFR1)IgG1-treated and TNFR1-deficient mice. J. Immunol. 157:3178-3182. 3. Joosten, L.A., Helsen, M.M., van de Loo, F.A., and van den Berg, W.B.
}

1996. Anticytokine treatment of established type II collagen-induced arthritis in DBA/1 mice. A comparative study using anti-TNF alpha, anti IL-1 alpha/beta and IL-1Ra. Arthritis Rheum. 39:797-809.

4. Takagi, N., et al. 1998. Blockage of interleukin- 6 receptor ameliorates joint disease in murine collagen-induced arthritis. Arthritis Rheum. 41:2117-2121.

5. Cook, A.D., Braine, E.L., Campbell, I.K., Rich, M.J., and Hamilton, J.A. 2001. Blockade of collagen-induced arthritis post-onset by antibody to granulocyte-macrophage colony-stimulating factor (GM-CSF): requirement for GM-CSF in the effector phase of disease. Arthritis Res. 3:293-298.

6. Moreland, L.W., et al. 1999. Etanercept therapy in rheumatoid arthritis. A randomized, controlled trial. Ann. Intern. Med. 130:478-486.

7. Campbell, I.K., O’Donnell, K., Lawlor, K.E., and Wicks, I.P. 2001. Severe inflammatory arthritis and lymphadenopathy in the absence of TNF. J. Clin. Invest. 107:1519-1527.

8. Alexander, W.S. 2002. Suppressors of cytokine signalling (SOCS) in the immune system. Nat. Rev. Immunol. 2:410-416.

9. Starr, R., et al. 1997. A family of cytokine-inducible inhibitors of signalling. Nature. 387:917-921.

10. Endo, T.A., et al. 1997. A new protein containing an $\mathrm{SH} 2$ domain that inhibits JAK kinases. Nature. 387:921-924.

11. Naka, T., et al. 1997. Structure and function of a new STAT-induced STAT inhibitor. Nature. 387:924-929.

12. Nicholson, S.E., et al. 2000 . Suppressor of cytokine signalling-3 preferentially binds to the SHP-2-binding site on the shared cytokine receptor subunit gp130. Proc. Natl. Acad. Sci. U. S. A. 97:6493-6498.

13. Morita, Y., et al. 2000. Signals transducers and activators of transcription (STAT)-induced STAT inhibitor-1 (SS-1)/suppressor of cytokine signalling-1 (SOCS-1) suppresses tumor necrosis factor $\alpha$-induced cell death in fibroblasts. Proc. Natl. Acad. Sci. U. S. A. 97:5405-5410.

14. Kinjyo, I., et al. 2002. SOCS1/JAB is a negative regulator of LPS-induced macrophage activation. Immunity. 17:583-591.

15. Nakagawa, R., et al. 2002. SOCS-1 participates in negative regulation of LPS responses. Immunity. 17:677-687.

16. Alonzi, T., et al. 1998. Interleukin 6 is required for the development of collagen-induced arthritis. J. Exp. Med. 187:461-468.

17. Sasai, M., et al. 1999. Delayed onset and reduced severity of collageninduced arthritis in interleukin-6-deficient mice. Arthritis Rheum. 42:1635-1643.

18. Campbell, I.K., et al. 1998. Protection from collagen-induced arthritis in granulocyte-macrophage colony stimulating factor-deficient mice. J. Immunol. 161:3639-3644.

19. Starr, R., et al. 1998. Liver degeneration and lymphoid deficiencies in mice lacking suppressor of cytokine signaling-1. Proc. Natl. Acad. Sci. U. S. A. 95:14395-14399.

20. Alexander, W.S., et al. 1999. SOCS1 is a critical inhibitor of interferon $\gamma$ signalling and prevents the potentially fatal neonatal actions of this cytokine. Cell. 98:597-608.

21. Staite, N.D., et al. 1990. Induction of an acute erosive monoarticular arthritis in mice by interleukin-1 and methylated bovine serum albumin. Arthritis Rheum. 33:253-260.

22. Lawlor, K.E., Campbell, I.K., O’Donnell, K., Wu, L., and Wicks, I.P. 2001. Molecular and cellular mediators of interleukin-1-dependent acute inflammatory arthritis. Arthritis Rheum. 44:442-450.

23. Elefanty, A.G., et al. 1998. Characterization of hematopoietic progenitor cells that express the transcription factor SCL, using a lacZ "knock-in" strategy. Proc. Natl. Acad. Sci. U. S. A. 95:11897-11902.

24. Cornish, A.L., et al. 2003. Suppressor of cytokine signalling-1 has IFN- $\gamma$ independent actions in T cell homeostasis. J. Immunol. 170:878-886.

25. Metcalf, D., et al. 2002. Polycystic kidneys and chronic inflammatory lesions are the delayed consequences of loss of the suppressor of cytokine signaling-1 (SOCS-1). Proc. Natl. Acad. Sci. U. S. A. 99:943-948.

26. Yang, Y.H., and Hamilton, J.A. 2001. Dependence of interleukin-1induced arthritis on granulocyte-macrophage colony stimulating factor. Arthritis Rheum. 44:111-119.

27. Ernst, M., et al. 2001. Defective gp130-mediated signal transducer and activator of transcription (STAT) signaling results in degenerative joint disease, gastrointestinal ulceration and failure of uterine implantation. J. Exp. Med. 194:189-203.

28. Sporri, B., Kovanen, P.E., Sasaki, A., Yoshimura, A., and Leonard, W.J. 2001. JAB/SOCS1/SSI-1 is an interleukin-2-induced inhibitor of IL-2 signalling. Blood. 97:221-226.

29. Matsuda, T., Yamamoto, T., Kishi, H., Yoshimura, A., and Muraguchi, A. 2000. SOCS-1 can suppress CD3ל-and Syk-mediated NF-AT activation in a non-lymphoid cell line. FEBS Lett. 472:235-240.

30. Shouda, T., et al. 2001. Induction of the cytokine signal regulator SOCS3/CIS3 as a therapeutic strategy for treating inflammatory arthritis. J. Clin. Invest. 108:1781-1788. doi:10.1172/JCI200113568. 